\title{
Obese Patients with Type 2 Diabetes: Outcomes After Laparoscopic Sleeve Gastrectomy
}

\section{German Viscido, MD; Veronica Gorodner, MD; Franco Jose Signorini, MD; A. Carolina Biasoni, MD; Luciano Navarro, MD; Graciela Rubin, MD; Lucio Obeide, MD and Federico Moser, MD \\ General Surgery and Diabetology and Nutrition- Hospital Privado Universitario de Córdoba- Argentina}

Background: Bariatric surgery is superior to medical treatment for type 2 diabetes mellitus (T2DM) control in obese patients. Reports in the literature have been mainly based on Roux-en-Y gastric bypass (RYGB) or adjustable gastric band. The aim of this study was to analyze mid- and long-term metabolic results after laparoscopic sleeve gastrectomy (LSG).

Methods: Obese patients with T2DM undergoing LSG were included in this study. Selection criteria for T2DM remission were: post-operatory fasting glucose (FG) level $\leq 100 \mathrm{mg} / \mathrm{LL}$ and hemoglobin $\mathrm{A} 1 \mathrm{c}(\mathrm{HbA} 1 \mathrm{c}) \leq 6 \%$ without medication.

Results: Between January 2009 and July 2016, 166 T2DM obese patients underwent LSG and completed $\geq 1$ year follow-up. There were 101 women (60.8\%; mean age $49.07 \pm 12.8$ years). Initial body mass index (BMI) was $46.44 \pm 7.68 \mathrm{~kg} / \mathrm{m} 2$. Mean time since T2DM diagnosis was 5.95 years $(1-28)$. Preoperative HbA1c was $7.53 \% \pm 0.97 \%$.

Before LSG, $75.3 \%(n=125)$ were receiving oral hypoglycemic agents, and $13.25 \%(n=22)$ insulin.

Mean follow-up was $65 \pm 10$ months.

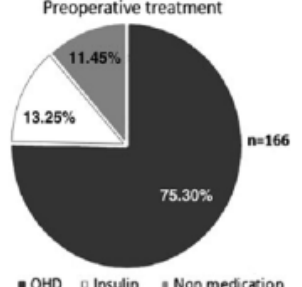

Complete T2DM remission was achieved in $78.3 \%, 76.2 \%$, and $71.4 \%$ at 1,3 , and $>5$ years respectively; in the long term, $7.2 \%$ attained partial remission, $10 \%$ improved, and $11.4 \%$ experienced recurrence of the disease.

Remission rate was significantly lower in patients under insulin therapy preoperatively, and in patients with T2DM diagnosed $>5$ years before consultation $(P=.0004$ and .0001 , respectively).

Table 1. Evolution at 1, 3, and 5 Years in Patients Showing Recurrence

\begin{tabular}{lccl}
\hline Variable & Patients with recurrence & Patients without recurrence & P \\
\hline Age (years) & $49.75 \pm 15.2$ & $48.53 \pm 14.4$ & $\mathrm{NS}$ \\
Disease duration (years) & $8.13 \pm 4.1$ & $5.75 \pm 2.9$ & $P=.0001$ \\
Preoperative BMI $\left(\mathrm{kg} / \mathrm{m}^{2}\right)$ & $39.6 \pm 5.82$ & $43 \pm 8.1 \mathrm{~kg} / \mathrm{m}^{2}$ & $\mathrm{NS}$ \\
Postoperative BMI at 5 years $\left(\mathrm{kg} / \mathrm{m}^{2}\right)$ & $34.32 \pm 4.17$ & $32.55 \pm 3.24$ & $\mathrm{NS}$ \\
\%EWL at 5 years & $54.83 \pm 15.2$ & $56.3 \pm 14.98$ & $\mathrm{NS}$ \\
Fasting glucose before LSG (mg/dL) & $170 \pm 56.95$ & $139.5 \pm 25.4$ & $P=.003$ \\
HbAlc before LSG (\%) & $8.56 \pm 2.18$ & $7.4 \pm 0.85 \%$ & $\mathrm{NS}$ \\
Outcomes at 1-year follow-up $(n=166)$ & $75 \%(n=6)$ complete & $79.5 \%(n=124)$ complete remission & \\
& remission & $9 \%(n=16)$ partial remission & \\
& $25 \%(n=2)$ improvement & $9 \%(n=14)$ improvement & \\
& & $2.5(n=4)$ without changes & \\
Outcomes at 3-year follow-up $(n=98)$ & $25 \%(n=2)$ complete & $80 \%(n=72)$ complete remission \\
& remission & $8 \%(n=7)$ partial remission \\
& $25 \%(n=2)$ partial remission & $8 \%(n=7)$ improvement \\
Outcomes at 5-year follow-up $(n=70)$ & $25 \%(n=2)$ improvement & $8 \%(n=4)$ without changes \\
& $100 \%(n=8)$ recurrence & $81 \%(n=50)$ complete remission \\
& & $8 \%(n=5)$ partial remission
\end{tabular}

\%EWL, percentage excess weight loss; LSG, laparoscopic sleeve gastrectomy
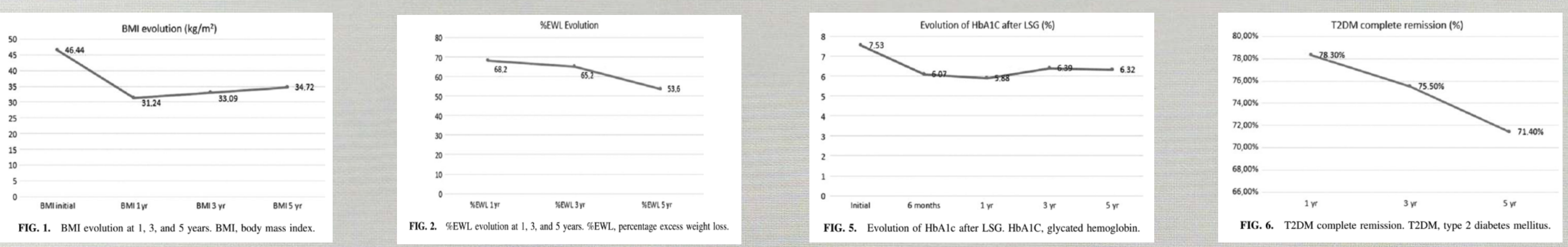

Conclusions: At mid- and long-term follow-up, T2DM control was satisfactory after LSG. Preoperative insulin therapy and T2DM duration $\geq 5$ years were predictors of less favorable outcomes. This work reveals the significant role of LSG in the treatment of T2DM in obese patients, since more than $70 \%$ support complete remission in the long term.

PROGRAMA DE CIRUEIA

$D E L A$ OBESIDAD 